\section{Urinary peritonitis caused by gangrenous cystitis}

\author{
BOUBAKER CHARRA • ABDELHAMID HACHIMI - MUSTAPHA SODKI • \\ HOUDA GUEDDARI • ABDELLATIF BENSLAMA • SAID MOTAOUAKKIL
}

\author{
BOUBAKER CHARRA • \\ ABDELHAMID HACHIMI • \\ MUSTAPHA SODKI • \\ HOUDA GUEDDARI • \\ ABDELLATIF BENSLAMA • \\ SAÎD MOTAOUAKKIL ( $\square$ ) \\ Medical Intensive Care Unit, \\ Ibn Rochd University Hospital \\ Casablanca 20 000, Morocco \\ Phone : 0021261155311 \\ E-mail : smotaouakkil@yahoo.fr
}

\begin{abstract}
We report a case of a young man who developed severe urinary sepsis, on the $21^{\text {st }}$ day of hospitalization (DH), which was treated with ciprofloxacin and gentamicin. On the $30^{\text {th }} \mathrm{DH}$, he developed bloodstream and urinary infections due to Acinetobacter baumannii which had been treated with colistin and rifampicin. On the $55^{\text {th }} \mathrm{DH}$, he developed urinary peritonitis and necrosis of the anterior and posterior bladder wall. Bilateral ureterostomy was performed. The patient was treated with colistin and imipenem. Peritoneal fluid culture yielded Enterobacter cloacae susceptible to imipenem. An enterocystoplasty was performed. The outcome was favourable.
\end{abstract}

Keywords: peritonitis, Acinetobacter baumannii, colistin, rifampicin

\section{Introduction}

Gangrenous cystitis is a rare condition that does not present with typical symptoms or clinical findings. This makes the diagnosis difficult and the prognosis poor. The authors report on a rare case of urinary peritonitis secondary to bladder necrosis.

\section{Case presentation}

We report the case of a 20- year- old man who underwent surgery in May 2005 for a cervical rachis injury. On the $21^{\text {st }} \mathrm{DH}$, the patient was admitted to the medical intensive care unit with severe sepsis. The patient presented with paraplegia and urinary retention for which a urethral catheter was placed. Urine culture yielded Proteus mirabilis susceptible to ciprofloxacin and gentamicin. Intravenous antibiotic therapy with ciprofloxacin (800 mg/day) and gentamicin (160 mg/day) was administered for two weeks. Progress was favourable. On the $30^{\text {th }} \mathrm{DH}$ in the medical intensive care unit, the patient devel- oped fever up to $39{ }^{\circ} \mathrm{C}$ and dropped his blood pressure $(70 / 40 \mathrm{mmHg})$. A. baumannii, susceptible to colistin, was isolated from blood and urine cultures. The patient was treated with colistin (2 million units, 3 times/day) and rifampicin (20 mg/kg/day) for two weeks with good recovery. In addition to antibiotic treatment, noradrenalin 1 $\mathrm{mg} / \mathrm{h}$ was administrated for two days. On the $55^{\text {th }} \mathrm{DH}$, the patient experienced vomiting, abdominal stiffness and fever up to $40^{\circ} \mathrm{C}$. An ultrasound showed an important peritoneal collection of fluid. Emergency surgery was performed. Surgical exploration revealed urinary peritonitis and necrosis of the anterior and posterior bladder wall. A bilateral ureterostomy was required. The patient was treated with colistin (2 million units, 3 times/day) and imipenem (2 gr/day). Enterobacter cloacae, susceptible to imipenem, were identified in the peritoneal fluid culture. The symptoms improved and an enterocystoplasty was performed.

\section{Discussion}

Prior to the use of antibiotics, gangrenous cystitis was a common problem attributed to vascular impairment, prolonged labour and infection secondary to urinary retention. (1) In the present case, a neurogenic bladder, antibiotic treatment and urinary infection may have been important etiological factors. The etiology of this disease is probably multifactorial and it is never possible to identify a unique cause. Several cases have been reported. Bragança reported a case of a diabetic male patient hospitalised with dehydration and diffuse abdominal pain. (2) Raza described a rare case of a colovesical fistula secondary to bladder necrosis. (3) The gangrenous cystitis presented with non- typical symptomatology apart from some urinary troubles, which are common to many other urological diseases. Emergency surgery is often performed without a preoperative defined diagnosis. The management of this condition is difficult. (4) Treatment should be early and aggressive. While regeneration of the bladder has been reported, the trigone was spared. (5) In our case a total cystectomy with enterocystoplasty was performed. This procedure allowed a good quality of life for the patient. 
Conclusion

Gangrenous cystitis is a rare condition and the etiopathogenesis is unknown.

Aggressive management should be instituted as soon as possible. We present a patient who survived severe urinary peritonitis caused by gangrene of the bladder. Due to entire bladder necrosis, preservation was not possible and hence total cystectomy, bilateral ureterocutaneostomy and abdominal drainage were performed.

\section{ACKNOWLEDGEMENTS}

The authors thank Prof. A Joual (urologist) for his participation in the management of the patient and Prof. M Benbachir (bacteriologist) for his revision of the paper.

\section{REFERENCES}

1. Striling WC, Hopkins GA. Gangrene of the bladder. Review of 207 cases and report of 2 personal cases. J Urol 1934;3:517-25.

2. Bragança N, Cruz T, Fonseca J, Faria M, Partidário J. Gangrenous cystitis. Acta Med Port 2001 May-Jun;14(3):357-9.

3. Raza A, Balsitis M, Hosie KB. Gangrenous cystitis: a rare cause of colovesical fistula. Postgrad Med J 1999;75:34-5.

4. Pollard SG. Colovesical fistula. Ann R Coll Surg 1987;69:163-5.

5. Devitt AT, Sethia KK. Gangrenous cystitis. Case report and review of the literature. J Urol 1993;149:1544-5. 